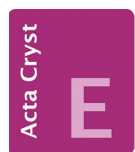

CRYSTALLOGRAPHIC COMMUNICATIONS

ISSN 2056-9890

Received 8 March 2017

Accepted 12 April 2017

Edited by H. Stoeckli-Evans, University of Neuchâtel, Switzerland

Keywords: crystal structure; phenothiazine; carbazole; carbaldehyde; $\mathrm{C}-\mathrm{H} \cdots \pi$ interactions; $\pi-\pi$ interactions.

CCDC reference: 1543611

Supporting information: this article has supporting information at journals.iucr.org/e

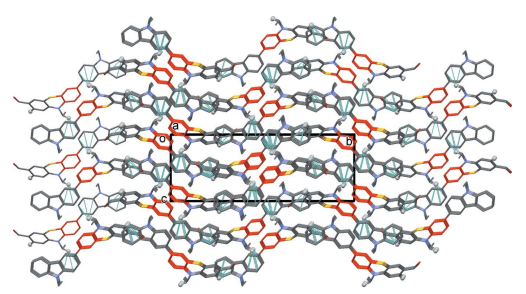

OPEN $\odot$ ACCESS

\section{Crystal structure of 10-ethyl-7-(9-ethyl-9H- carbazol-3-yl)-10H-phenothiazine-3-carbaldehyde}

\author{
Vairavan Mahalakshmi, ${ }^{a}$ Siddan Gouthaman, ${ }^{\mathrm{b}}$ Madurai Sugunalakshmi, ${ }^{\mathrm{b}}$ Srinivasan \\ Bargavi $^{\mathrm{a}}$ and Srinivasakannan Lakshmi ${ }^{\mathrm{a} *}$
}

\begin{abstract}
${ }^{\mathbf{a}}$ Department of Physics, S.D.N.B. Vaishnav College for Women, Chromepet, Chennai 600 044, India, and ${ }^{\mathbf{b}}$ Industrial Chemistry Polymer Division, CSIR Central Leather Research Institute, Chennai 600 020, India. *Correspondence e-mail: lakssdnbvc@gmail.com
\end{abstract}

The title compound, $\mathrm{C}_{29} \mathrm{H}_{24} \mathrm{~N}_{2} \mathrm{OS}$, contains a phenothiazine moiety linked to a planar carbazole unit (r.m.s. deviation $=0.029 \AA$ ) by a $\mathrm{C}-\mathrm{C}$ single bond. The phenothiazine moiety possesses a typical non-planar butterfly structure with a fold angle of $27.36(9)^{\circ}$ between the two benzene rings. The dihedral angle between the mean planes of the carbazole and phenothiazine units is $27.28(5)^{\circ}$. In the crystal, molecules stack in pairs along the $c$-axis direction, linked by offset $\pi-\pi$ interactions [intercentroid distance $=3.797$ (1) $\AA$ ] . There are $\mathrm{C}-\mathrm{H} \cdots \pi$ interactions present linking these dimers to form a three-dimensional structure.

\section{Chemical context}

Phenothiazine, related to the thiazine class of heterocyclic compounds, is very important as it occurs in various antipsychotic drugs. Phenothiazine derivatives have been used in dye-sensitized solar cells to study the effect of conjugated linkers on device performance (Kim et al., 2011; Hagfeldt et al., 2010). One phenothiazine derivative (MCDP) is used to measure the activity of monoamine oxidase in blood (Fujii et al., 1993). They are also used as neuroleptics, sedatives, analgesics, anti-emetics and antihistamines (Harris \& Klein, 1987). Triflupromazine phenothiazine hydrochloride is one of the most potent tranquilizer drug molecules (Phelps \& Cordes, 1974). The phenothiazine derivative thiethylperazine has the properties of an anti-emetic and is widely used for the control of post-operative vomiting, in radiation therapy and vomiting associated with malignant disease (McDowell, 1970, 1978). $\mathrm{N}$-Alkylamino carbazoles show significant anti-convulsant and diuretic activity (Shoeb et al., 1973). One of them, rimcazole, is a well known anti-pyretic and neuroleptic agent (Ferris et al., 1986). In view of this interest, we have synthesized the title phenothiazine derivative and report herein on its crystal structure.

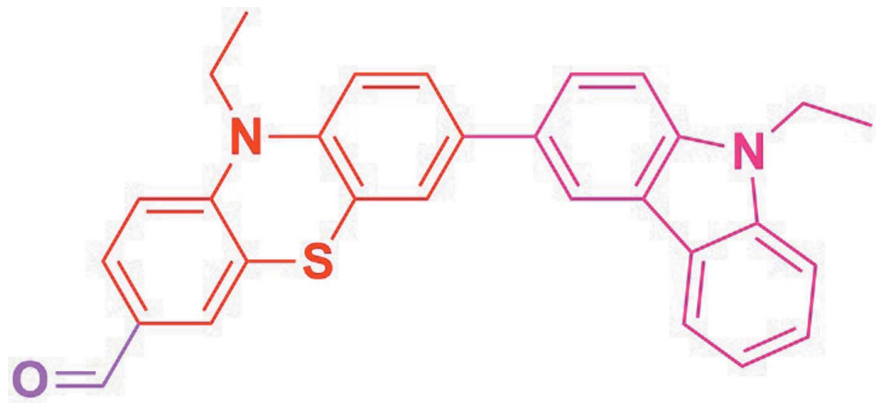




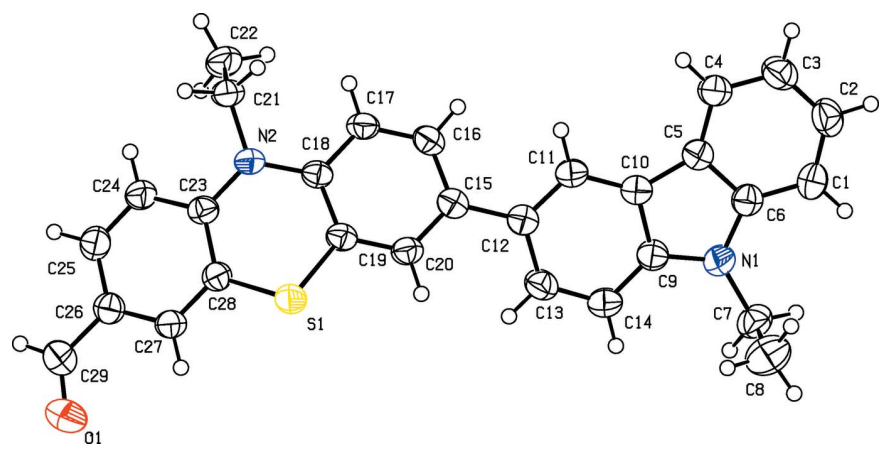

Figure 1

The molecular structure of the title compound, with the atom labelling and displacement ellipsoids drawn at the $50 \%$ probability level.

\section{Structural commentary}

In the title compound, the phenothiazine moiety has a nonplanar butterfly structure (Fig. 1), similar to that observed for 10-methyl-10H-phenothiazine (Malikireddy et al., 2016). The central six-membered ring (N2/C18/C19/S1/C28/C23) adopts a boat conformation [puckering parameters are: $Q_{\mathrm{T}}=$ 0.4567 (16) $\left.\AA, \theta=102.8(2)^{\circ}, \varphi=182.8(2)^{\circ}\right]$. The fold angle of $27.36(9)^{\circ}$ between the two benzene rings of this moiety compares well with the values reported for similar compounds (CSD; Groom et al., 2016). The dihedral angle between the planes of the two benzene rings of the carbazole ring is $2.94(10)^{\circ}$ and the dihedral angle between the mean planes of the carbazole and phenothiazine ring systems is $27.28(5)^{\circ}$. The aldehyde group is almost coplanar with the benzene ring to which it is attached, the $\mathrm{C} 27-\mathrm{C} 26-\mathrm{C} 29-\mathrm{O} 1$ torsion angle
Table 1

Hydrogen-bond geometry $\left(\AA,^{\circ}\right)$.

$\mathrm{Cg} 3$ and $\mathrm{Cg} 4$ are the centroids of the $\mathrm{C} 1-\mathrm{C} 6$ and $\mathrm{C} 9-\mathrm{C} 14$ rings, respectively.

\begin{tabular}{lllll}
\hline$D-\mathrm{H} \cdots A$ & $D-\mathrm{H}$ & $\mathrm{H} \cdots A$ & $D \cdots A$ & $D-\mathrm{H} \cdots A$ \\
\hline $\mathrm{C} 21-\mathrm{H} 21 A \cdots C g 4^{\mathrm{i}}$ & 0.97 & 2.95 & $3.596(2)$ & 125 \\
$\mathrm{C} 25-\mathrm{H} 25 \cdots C g 3^{\mathrm{ii}}$ & 0.93 & 2.96 & $3.558(2)$ & 123
\end{tabular}

Symmetry codes: (i) $-x+1,-y,-z+2$; (ii) $-x+\frac{3}{2}, y+\frac{1}{2},-z+\frac{3}{2}$.

being $0.9(4)^{\circ}$. The ethyl groups protrude out of the planes of the carbazole and phenothiazine skeletons, as indicated by the torsion angles $\mathrm{C} 6-\mathrm{N} 1-\mathrm{C} 7-\mathrm{C} 8=87.7(3)^{\circ}$ and $\mathrm{C} 23-\mathrm{N} 2-$ $\mathrm{C} 21-\mathrm{C} 22=-83.2(2)^{\circ}$.

\section{Supramolecular features}

In the crystal, inversion-related molecules stack in pairs along the $c$-axis direction, linked by offset $\pi-\pi$ interactions $\left[C g 5 \cdots C g 5^{\mathrm{i}}=3.7965(11) \AA\right.$, interplanar distance = 3.5133 (8) $\AA$, slippage $=1.439 \AA, C g 5$ is the centroid of the C15-C20 ring; symmetry code: (i) $-x+1,-y,-z+2]$. There are also $\mathrm{C}-\mathrm{H} \cdots \pi$ interactions present linking these dimers to form a three-dimensional structure (Table 1 and Fig. 2).

\section{Database survey}

A search of the Cambridge Structural Database (CSD, Version 5.38, update February 2017; Groom et al., 2016) for compounds containing either a phenothiazine, carbazole or carbaldehyde unit gave 433 hits for compounds containing a

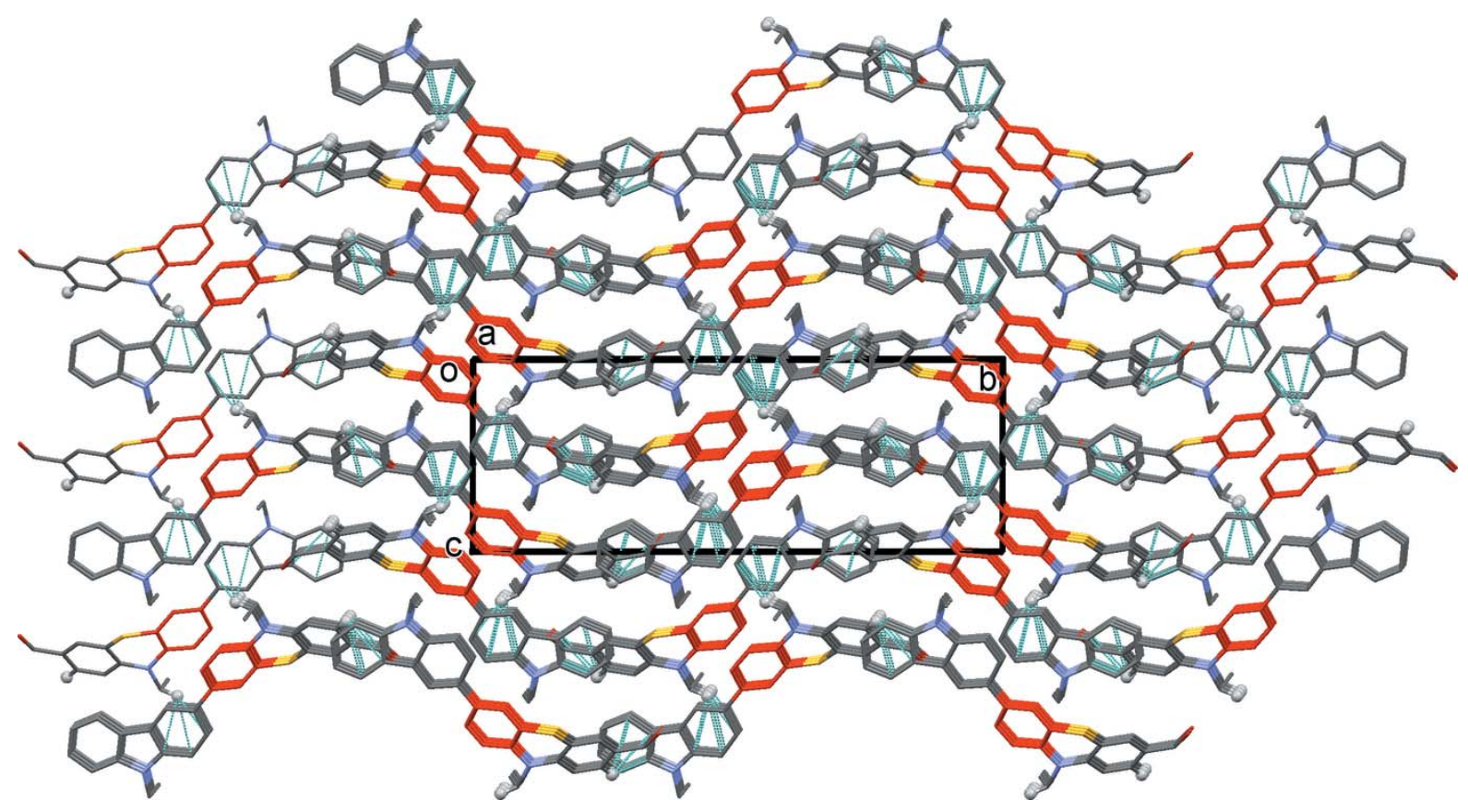

Figure 2

The crystal packing of the title compound, viewed along the $a$ axis. The C15-C20 rings linked by $\pi-\pi$ interactions are shown in red. For clarity, only the $\mathrm{H}$ atoms (grey balls) involved in the $\mathrm{C}-\mathrm{H} \cdots \pi$ interactions (dashed lines; see Table 1 ) have been included. 
Table 2

Experimental details.

\begin{tabular}{|c|c|}
\hline \multicolumn{2}{|l|}{ Crystal data } \\
\hline Chemical formula & $\mathrm{C}_{29} \mathrm{H}_{24} \mathrm{~N}_{2} \mathrm{OS}$ \\
\hline$M_{\mathrm{r}}$ & 448.56 \\
\hline Crystal system, space group & Monoclinic, $P 2_{1} / n$ \\
\hline Temperature $(\mathrm{K})$ & 296 \\
\hline$a, b, c(\AA)$ & $9.4677(6), 25.7169(13), 9.5704(5)$ \\
\hline$\beta\left(^{\circ}\right)$ & $103.681(2)$ \\
\hline$V\left(\AA^{3}\right)$ & $2264.1(2)$ \\
\hline$Z$ & 4 \\
\hline Radiation type & Мo $K \alpha$ \\
\hline$\mu\left(\mathrm{mm}^{-1}\right)$ & 0.17 \\
\hline Crystal size $(\mathrm{mm})$ & $0.30 \times 0.25 \times 0.20$ \\
\hline \multicolumn{2}{|l|}{ Data collection } \\
\hline Diffractometer & Bruker Kappa APEXII CCD \\
\hline Absorption correction & $\begin{array}{l}\text { Multi-scan ( } S A D A B S \text {; Bruker, } \\
\text { 2004) }\end{array}$ \\
\hline$T_{\min }, T_{\max }$ & $0.677,0.745$ \\
\hline $\begin{array}{l}\text { No. of measured, independent and } \\
\text { observed }[I>2 \sigma(I)] \text { reflections }\end{array}$ & 25526, 3981, 2974 \\
\hline$R_{\text {int }}$ & 0.036 \\
\hline$(\sin \theta / \lambda)_{\max }\left(\AA^{-1}\right)$ & 0.595 \\
\hline \multicolumn{2}{|l|}{ Refinement } \\
\hline$R\left[F^{2}>2 \sigma\left(F^{2}\right)\right], w R\left(F^{2}\right), S$ & $0.040,0.107,1.03$ \\
\hline No. of reflections & 3981 \\
\hline No. of parameters & 300 \\
\hline $\mathrm{H}$-atom treatment & H-atom parameters constrained \\
\hline$\Delta \rho_{\max }, \Delta \rho_{\min }\left(\mathrm{e} \AA^{-3}\right)$ & $0.18,-0.20$ \\
\hline
\end{tabular}

Computer programs: APEX2 and SAINT (Bruker, 2004), SHELXS97 (Sheldrick, 2008), SHELXL2014 (Sheldrick, 2015), PLATON (Spek, 2009), Mercury (Macrae et al., 2008) and publCIF (Westrip, 2010).

phenothiazine unit, and 2293 hits for compounds containing a carbazole unit. Out of these entries, six compounds were found to possess both phenothiazine and carbazole ring systems, and one compound contains all three units, phenothiazine, carbazole and a carbaldehyde unit, but with the carbazole unit linked directly to the $\mathrm{N}$ atom of the phenothiazine unit, viz. 10-(9-hexyl-9H-carbazol-3yl)-10H-phenothiazine-3-carbaldehyde (IWABUF; Karuppasamy et al., 2017).

\section{Synthesis and crystallization}

To a mixture of 7-bromo-10-ethyl-10H-phenothiazine-3carbaldehyde (3 g, $0.0089 \mathrm{~mol}), \quad 9$-ethyl-9H-carbazole-3boronic acid pinnacol ester (3.17 g, 1.1 eq.), $\mathrm{Pd}\left(\mathrm{PPh}_{3}\right)_{4}$ (518 mg, 5\% mol) and $\mathrm{K}_{2} \mathrm{CO}_{3}(2.48 \mathrm{~g}, 2$ eq.) under high vacuum was added a mixture of toluene:water (2:1). The resulting mixture was heated to reflux under an $\mathrm{N}_{2}$ atmosphere for $c a 24 \mathrm{~h}$. On completion of the reaction (monitored by TLC), it was quenched by addition of saturated doubledistilled $\mathrm{H}_{2} \mathrm{O}$ and extracted with dichloromethane. The organic phases were collected and washed with brine and dried over anhydrous $\mathrm{Na}_{2} \mathrm{SO}_{4}$ and then concentrated. The product was purified by column chromatography on silica gel using ethyl acetate: $n$-hexane $(12: 88, v / v)$ as eluent, to give the title compound as a pale-yellow crystalline solid (yield $80 \%$ ). It was characterized by ${ }^{1} \mathrm{H}$ NMR, ${ }^{13} \mathrm{C}$ NMR, IR and ESIMASS. Brown block-like crystals of the title compound were obtained by slow evaporation at room temperature of a solution in dichloromethane and acetonitrile $(1: 1 \mathrm{v} / \mathrm{v})$.

\section{Refinement}

Crystal data, data collection and structure refinement details are summarized in Table 2. All $\mathrm{H}$ atoms were placed in calculated positions and refined using a riding-model approximation: $\mathrm{C}-\mathrm{H}=0.93-0.98 \AA$ with $U_{\text {iso }}(\mathrm{H})=1.5 U_{\text {eq }}(\mathrm{C}-$ methyl) and $1.2 U_{\text {eq }}(\mathrm{C})$ for other $\mathrm{H}$ atoms.

\section{Acknowledgements}

The authors thank the single-crystal XRD facility, SAIF IIT Madras, Chennai, for the data collection.

\section{References}

Bruker (2004). APEX2, SAINT and SADABS. Bruker AXS Inc., Madison, Wisconsin, USA.

Ferris, R. M., White, H. L., Tang, F. L. M., Russell, A. \& Harfenist, M. (1986). Drug Dev. Res. 9, 171-188.

Fujii, I., Hirayama, N. \& Miike, A. (1993). Acta Cryst. C49, 1540-1541.

Groom, C. R., Bruno, I. J., Lightfoot, M. P. \& Ward, S. C. (2016). Acta Cryst. B72, 171-179.

Hagfeldt, A., Boschloo, G., Sun, L.-C., Kloo, L. \& Pettersson, H. (2010). Chem. Rev. 110, 6595-6663.

Harris, J. E. \& Klein, C. L. (1987). Acta Cryst. C43, 1737-1739.

Karuppasamy, A., Krishnan, K. G., Pillai, M. P. V. \& Ramalingan, C. (2017). J. Mol. Struct. 1128, 674-684.

Kim, S. H., Kim, H. W., Sakong, C., Namgoong, J., Park, S. W., Ko, M. J., Lee, C. H., Lee, W. I. \& Kim, J. P. (2011). Org. Lett. 13, 57845787.

Macrae, C. F., Bruno, I. J., Chisholm, J. A., Edgington, P. R., McCabe, P., Pidcock, E., Rodriguez-Monge, L., Taylor, R., van de Streek, J. \& Wood, P. A. (2008). J. Appl. Cryst. 41, 466-470.

Malikireddy, P., Siddan, G., Madurai, S., Chandramouleeswaran, S. \& Srinivasakannan, L. (2016). IUCrData, 1, x161299.

McDowell, J. J. H. (1970). Acta Cryst. B26, 954-964.

McDowell, J. J. H. (1978). Acta Cryst. B34, 686-689.

Phelps, D. W. \& Cordes, A. W. (1974). Acta Cryst. B30, 2812-2816.

Sheldrick, G. M. (2008). Acta Cryst. A64, 112-122.

Sheldrick, G. M. (2015). Acta Cryst. C71, 3-8.

Shoeb, A., Anwer, F., Kapil, R. S., Popli, S. P., Dua, P. \& Dhawan, B. N. (1973). J. Med. Chem. 16, 425-427.

Spek, A. L. (2009). Acta Cryst. D65, 148-155.

Westrip, S. P. (2010). J. Appl. Cryst. 43, 920-925. 


\section{supporting information}

Acta Cryst. (2017). E73, 726-728 [https://doi.org/10.1107/S2056989017005540]

\section{Crystal structure of 10-ethyl-7-(9-ethyl-9H-carbazol-3-yl)-10H-phenothiazine-3- carbaldehyde}

\section{Vairavan Mahalakshmi, Siddan Gouthaman, Madurai Sugunalakshmi, Srinivasan Bargavi and}

\section{Srinivasakannan Lakshmi}

\section{Computing details}

Data collection: APEX2 (Bruker, 2004); cell refinement: SAINT (Bruker, 2004); data reduction: SAINT (Bruker, 2004); program(s) used to solve structure: SHELXS97 (Sheldrick, 2008); program(s) used to refine structure: SHELXL2014 (Sheldrick, 2015); molecular graphics: PLATON (Spek, 2009) and Mercury (Macrae et al., 2008); software used to prepare material for publication: publCIF (Westrip, 2010).

10-Ethyl-7-(9-ethyl-9H-carbazol-3-yl)-10H-phenothiazine-3-carbaldehyde

Crystal data

$\mathrm{C}_{29} \mathrm{H}_{24} \mathrm{~N}_{2} \mathrm{OS}$

$M_{r}=448.56$

Monoclinic, $P 2_{1} / n$

$a=9.4677(6) \AA$

$b=25.7169(13) \AA$

$c=9.5704(5) \AA$

$\beta=103.681(2)^{\circ}$

$V=2264.1(2) \AA^{3}$

$Z=4$

\section{Data collection}

Bruker Kappa APEXII CCD diffractometer

Bruker axs kappa axes2 CCD scans

Absorption correction: multi-scan

(SADABS; Bruker, 2004)

$T_{\text {min }}=0.677, T_{\max }=0.745$

25526 measured reflections

\section{Refinement}

Refinement on $F^{2}$

Least-squares matrix: full

$R\left[F^{2}>2 \sigma\left(F^{2}\right)\right]=0.040$

$w R\left(F^{2}\right)=0.107$

$S=1.03$

3981 reflections

300 parameters

0 restraints
$F(000)=944$

$D_{\mathrm{x}}=1.316 \mathrm{Mg} \mathrm{m}^{-3}$

Mo $K \alpha$ radiation, $\lambda=0.71073 \AA$

Cell parameters from 6254 reflections

$\theta=2.4-23.8^{\circ}$

$\mu=0.17 \mathrm{~mm}^{-1}$

$T=296 \mathrm{~K}$

Block, brown

$0.30 \times 0.25 \times 0.20 \mathrm{~mm}$

3981 independent reflections

2974 reflections with $I>2 \sigma(I)$

$R_{\text {int }}=0.036$

$\theta_{\max }=25.0^{\circ}, \theta_{\min }=2.3^{\circ}$

$h=-11 \rightarrow 9$

$k=-30 \rightarrow 30$

$l=-11 \rightarrow 11$

Primary atom site location: structure-invariant direct methods

Secondary atom site location: difference Fourier map

Hydrogen site location: inferred from neighbouring sites

$\mathrm{H}$-atom parameters constrained 
$w=1 /\left[\sigma^{2}\left(F_{\mathrm{o}}^{2}\right)+(0.0455 P)^{2}+0.8313 P\right]$

where $P=\left(F_{\mathrm{o}}^{2}+2 F_{\mathrm{c}}^{2}\right) / 3$

$(\Delta / \sigma)_{\max }=0.004$

$$
\Delta \rho_{\max }=0.18 \text { e } \AA^{-3}
$$

\section{Special details}

Geometry. All esds (except the esd in the dihedral angle between two 1.s. planes) are estimated using the full covariance matrix. The cell esds are taken into account individually in the estimation of esds in distances, angles and torsion angles; correlations between esds in cell parameters are only used when they are defined by crystal symmetry. An approximate (isotropic) treatment of cell esds is used for estimating esds involving 1.s. planes.

Fractional atomic coordinates and isotropic or equivalent isotropic displacement parameters $\left(\AA^{2}\right)$

\begin{tabular}{|c|c|c|c|c|}
\hline & $x$ & $y$ & $z$ & $U_{\text {iso }} * / U_{\text {eq }}$ \\
\hline $\mathrm{S} 1$ & $0.49912(6)$ & $0.15680(2)$ & $0.93196(6)$ & $0.04721(18)$ \\
\hline $\mathrm{N} 2$ & $0.78333(17)$ & $0.11025(6)$ & $1.10224(16)$ & $0.0385(4)$ \\
\hline $\mathrm{C} 5$ & $0.3217(2)$ & $-0.16204(8)$ & $0.5560(2)$ & $0.0395(5)$ \\
\hline C15 & $0.5175(2)$ & $0.01031(7)$ & $0.7928(2)$ & $0.0385(5)$ \\
\hline $\mathrm{C} 12$ & $0.4282(2)$ & $-0.02290(8)$ & $0.6785(2)$ & $0.0393(5)$ \\
\hline $\mathrm{C} 10$ & $0.3469(2)$ & $-0.10671(8)$ & $0.5781(2)$ & $0.0380(5)$ \\
\hline $\mathrm{C} 17$ & $0.7263(2)$ & $0.02401(8)$ & $0.9923(2)$ & $0.0423(5)$ \\
\hline H17 & 0.8081 & 0.0101 & 1.0547 & $0.051^{*}$ \\
\hline $\mathrm{C} 18$ & $0.6941(2)$ & $0.07646(7)$ & $1.0030(2)$ & $0.0362(5)$ \\
\hline $\mathrm{C} 23$ & $0.7869(2)$ & $0.16369(7)$ & $1.0771(2)$ & $0.0374(5)$ \\
\hline $\mathrm{C} 21$ & $0.9010(2)$ & $0.08767(8)$ & $1.2138(2)$ & $0.0443(5)$ \\
\hline $\mathrm{H} 21 \mathrm{~A}$ & 0.8654 & 0.0561 & 1.2489 & $0.053 *$ \\
\hline $\mathrm{H} 21 \mathrm{~B}$ & 0.9253 & 0.1119 & 1.2936 & $0.053 *$ \\
\hline C28 & $0.6646(2)$ & $0.18995(7)$ & $0.9936(2)$ & $0.0381(5)$ \\
\hline C16 & $0.6397(2)$ & -0.00788 & $0.8909(2)$ & $0.0424(5)$ \\
\hline H16 & 0.6642 & -0.0428 & 0.8884 & $0.051^{*}$ \\
\hline N1 & $0.1914(2)$ & $-0.11924(7)$ & $0.35912(18)$ & $0.0470(5)$ \\
\hline C11 & $0.4296(2)$ & $-0.07678(8)$ & $0.6882(2)$ & $0.0394(5)$ \\
\hline H11 & 0.4864 & -0.0929 & 0.7692 & $0.047^{*}$ \\
\hline $\mathrm{C} 26$ & $0.7923(2)$ & $0.27184(8)$ & $1.0287(2)$ & $0.0438(5)$ \\
\hline $\mathrm{C} 27$ & $0.6683(2)$ & $0.24282(8)$ & $0.9705(2)$ & $0.0436(5)$ \\
\hline $\mathrm{H} 27$ & 0.5866 & 0.2593 & 0.9152 & $0.052 *$ \\
\hline C9 & $0.2620(2)$ & $-0.08213(8)$ & $0.4554(2)$ & $0.0420(5)$ \\
\hline C19 & $0.5669(2)$ & $0.09442(7)$ & $0.9097(2)$ & $0.0377(5)$ \\
\hline $\mathrm{C} 20$ & $0.4834(2)$ & $0.06242(7)$ & $0.8066(2)$ & $0.0403(5)$ \\
\hline $\mathrm{H} 20$ & 0.4014 & 0.0762 & 0.7442 & $0.048 *$ \\
\hline $\mathrm{C} 14$ & $0.2576(2)$ & $-0.02860(8)$ & $0.4439(2)$ & $0.0498(6)$ \\
\hline H14 & 0.1999 & -0.0124 & 0.3635 & $0.060^{*}$ \\
\hline $\mathrm{O} 1$ & $0.7031(2)$ & $0.35389(7)$ & $0.9339(2)$ & $0.0900(7)$ \\
\hline C13 & $0.3407(2)$ & $0.00008(8)$ & $0.5540(2)$ & $0.0482(5)$ \\
\hline H13 & 0.3392 & 0.0361 & 0.5463 & $0.058 *$ \\
\hline $\mathrm{C} 24$ & $0.9103(2)$ & $0.19363(8)$ & $1.1355(2)$ & $0.0444(5)$ \\
\hline $\mathrm{H} 24$ & 0.9926 & 0.1776 & 1.1913 & $0.053 *$ \\
\hline C6 & $0.2262(2)$ & $-0.16776(8)$ & $0.4193(2)$ & $0.0420(5)$ \\
\hline $\mathrm{C} 29$ & 0.8000 & $0.32760(9)$ & $1.0036(3)$ & $0.0579(6)$ \\
\hline $\mathrm{H} 29$ & 0.8870 & 0.3443 & 1.0448 & $0.069 *$ \\
\hline
\end{tabular}




$\begin{array}{lllll}\text { C4 } & 0.3684(2) & -0.20599(8) & 0.6386(2) & 0.0474(5) \\ \text { H4 } & 0.4303 & -0.2030 & 0.7295 & 0.057^{*} \\ \text { C7 } & 0.0799(2) & -0.10852(9) & 0.2300(2) & 0.0535(6) \\ \text { H7A } & 0.0743 & -0.1375 & 0.1641 & 0.064^{*} \\ \text { H7B } & 0.1074 & -0.0779 & 0.1836 & 0.064^{*} \\ \text { C25 } & 0.9118(2) & 0.24642(8) & 1.1116(2) & 0.0474(5) \\ \text { H25 } & 0.9951 & 0.2654 & 1.1521 & 0.057^{*} \\ \text { C1 } & 0.1828(2) & -0.21668(8) & 0.3631(2) & 0.0509(6) \\ \text { H1 } & 0.1226 & -0.2204 & 0.2716 & 0.061^{*} \\ \text { C22 } & 1.0392(2) & 0.07482(9) & 1.1657(2) & 0.0561(6) \\ \text { H22A } & 1.0222 & 0.0452 & 1.1028 & 0.084^{*} \\ \text { H22B } & 1.1159 & 0.0671 & 1.2483 & 0.084^{*} \\ \text { H22C } & 1.0665 & 0.1041 & 1.1156 & 0.084^{*} \\ \text { C3 } & 0.3213(3) & -0.25401(9) & 0.5835(3) & 0.0553(6) \\ \text { H3 } & 0.3502 & -0.2836 & 0.6389 & 0.066^{*} \\ \text { C2 } & 0.2315(2) & -0.25924(9) & 0.4470(3) & 0.0560(6) \\ \text { H2 } & 0.2037 & -0.2923 & 0.4116 & 0.067^{*} \\ \text { C8 } & -0.0676(3) & -0.09976(12) & 0.2586(3) & 0.0787(8) \\ \text { H8A } & -0.0938 & -0.1292 & 0.3088 & 0.118^{*} \\ \text { H8B } & -0.1380 & -0.0954 & 0.1690 & 0.118^{*} \\ \text { H8C } & -0.0654 & -0.0691 & 0.3163 & 0.118^{*}\end{array}$

Atomic displacement parameters $\left(\AA^{2}\right)$

\begin{tabular}{|c|c|c|c|c|c|c|}
\hline & $U^{11}$ & $U^{22}$ & $U^{33}$ & $U^{12}$ & $U^{13}$ & $U^{23}$ \\
\hline $\mathrm{S} 1$ & $0.0356(3)$ & $0.0397(3)$ & $0.0623(4)$ & $0.0083(2)$ & $0.0034(3)$ & $-0.0034(3)$ \\
\hline $\mathrm{N} 2$ & $0.0359(10)$ & $0.0368(9)$ & $0.0398(9)$ & $0.0067(7)$ & $0.0032(8)$ & $0.0026(7)$ \\
\hline $\mathrm{C} 5$ & $0.0376(12)$ & 0.0409 (11) & $0.0417(11)$ & $0.0010(9)$ & $0.0125(9)$ & $0.0006(9)$ \\
\hline C15 & 0.0387 (12) & $0.0376(11)$ & $0.0420(11)$ & $0.0009(9)$ & $0.0150(9)$ & $0.0031(9)$ \\
\hline $\mathrm{C} 12$ & $0.0394(12)$ & 0.0407 (11) & $0.0399(11)$ & $0.0002(9)$ & $0.0133(9)$ & $0.0028(9)$ \\
\hline C10 & $0.0378(11)$ & $0.0404(11)$ & $0.0376(11)$ & $0.0033(9)$ & $0.0123(9)$ & $0.0023(9)$ \\
\hline $\mathrm{C} 17$ & $0.0382(12)$ & $0.0389(11)$ & 0.0473 (12) & $0.0075(9)$ & $0.0052(10)$ & $0.0064(9)$ \\
\hline $\mathrm{C} 18$ & 0.0345 (11) & $0.0367(11)$ & 0.0388 (11) & $0.0034(9)$ & $0.0114(9)$ & $0.0044(9)$ \\
\hline $\mathrm{C} 23$ & 0.0377 (11) & 0.0403 (11) & $0.0346(10)$ & $0.0066(9)$ & $0.0093(9)$ & $-0.0006(9)$ \\
\hline $\mathrm{C} 21$ & $0.0448(13)$ & $0.0438(12)$ & $0.0404(11)$ & $0.0086(10)$ & $0.0023(10)$ & $0.0043(9)$ \\
\hline $\mathrm{C} 28$ & $0.0386(12)$ & $0.0393(11)$ & $0.0358(11)$ & $0.0042(9)$ & $0.0072(9)$ & $-0.0033(9)$ \\
\hline $\mathrm{C} 16$ & $0.0438(13)$ & 0.0337 (11) & 0.0503 (12) & $0.0038(9)$ & $0.0124(10)$ & $0.0026(9)$ \\
\hline N1 & $0.0502(11)$ & $0.0465(10)$ & $0.0401(9)$ & $-0.0018(8)$ & $0.0023(8)$ & $0.0029(8)$ \\
\hline $\mathrm{C} 11$ & 0.0397 (12) & $0.0425(12)$ & $0.0362(11)$ & $0.0041(9)$ & $0.0094(9)$ & $0.0048(9)$ \\
\hline $\mathrm{C} 26$ & $0.0512(13)$ & 0.0419 (12) & $0.0379(11)$ & $0.0036(10)$ & $0.0096(10)$ & $-0.0023(9)$ \\
\hline $\mathrm{C} 27$ & 0.0469 (13) & 0.0407 (12) & $0.0394(11)$ & $0.0081(10)$ & $0.0026(10)$ & $0.0002(9)$ \\
\hline C9 & 0.0457 (13) & $0.0424(12)$ & $0.0380(11)$ & $-0.0021(10)$ & $0.0103(10)$ & $0.0034(9)$ \\
\hline C19 & $0.0353(11)$ & $0.0365(11)$ & $0.0418(11)$ & $0.0047(9)$ & $0.0104(9)$ & $0.0034(9)$ \\
\hline $\mathrm{C} 20$ & $0.0348(11)$ & $0.0417(12)$ & $0.0429(11)$ & $0.0041(9)$ & $0.0060(9)$ & $0.0051(9)$ \\
\hline $\mathrm{C} 14$ & $0.0568(14)$ & $0.0477(13)$ & $0.0408(12)$ & $0.0019(11)$ & $0.0032(11)$ & $0.0104(10)$ \\
\hline $\mathrm{O} 1$ & $0.0925(15)$ & $0.0504(11)$ & $0.1099(16)$ & $0.0041(10)$ & $-0.0101(13)$ & $0.0203(11)$ \\
\hline C13 & 0.0585 (15) & $0.0379(11)$ & $0.0474(12)$ & $0.0016(10)$ & $0.0109(11)$ & $0.0065(10)$ \\
\hline $\mathrm{C} 24$ & $0.0384(12)$ & $0.0436(12)$ & $0.0476(12)$ & $0.0065(10)$ & $0.0034(10)$ & $-0.0025(10)$ \\
\hline
\end{tabular}




\begin{tabular}{lllllll}
\hline C6 & $0.0371(12)$ & $0.0443(12)$ & $0.0453(12)$ & $-0.0021(9)$ & $0.0112(9)$ & $0.0009(10)$ \\
C29 & $0.0669(17)$ & $0.0443(13)$ & $0.0594(15)$ & $-0.0015(12)$ & $0.0087(13)$ & $0.0006(12)$ \\
C4 & $0.0461(13)$ & $0.0454(13)$ & $0.0497(12)$ & $0.0033(10)$ & $0.0095(10)$ & $0.0051(10)$ \\
C7 & $0.0557(15)$ & $0.0612(14)$ & $0.0395(12)$ & $-0.0032(11)$ & $0.0035(11)$ & $0.0043(11)$ \\
C25 & $0.0450(13)$ & $0.0477(12)$ & $0.0479(12)$ & $-0.0027(10)$ & $0.0076(10)$ & $-0.0064(10)$ \\
C1 & $0.0441(13)$ & $0.0527(14)$ & $0.0539(13)$ & $-0.0096(11)$ & $0.0074(11)$ & $-0.0053(11)$ \\
C22 & $0.0432(13)$ & $0.0576(14)$ & $0.0610(15)$ & $0.0145(11)$ & $-0.0006(11)$ & $-0.0043(12)$ \\
C3 & $0.0539(15)$ & $0.0431(13)$ & $0.0692(16)$ & $-0.0002(11)$ & $0.0152(13)$ & $0.0084(11)$ \\
C2 & $0.0500(14)$ & $0.0444(13)$ & $0.0740(17)$ & $-0.0101(11)$ & $0.0154(13)$ & $-0.0058(12)$ \\
C8 & $0.0529(17)$ & $0.099(2)$ & $0.0816(19)$ & $0.0087(15)$ & $0.0108(14)$ & $0.0031(17)$ \\
\hline
\end{tabular}

Geometric parameters $\left(\hat{A},{ }^{o}\right)$

\begin{tabular}{|c|c|c|c|}
\hline $\mathrm{S} 1-\mathrm{C} 28$ & $1.759(2)$ & $\mathrm{C} 26-\mathrm{C} 27$ & $1.392(3)$ \\
\hline $\mathrm{S} 1-\mathrm{C} 19$ & 1.7595 (19) & $\mathrm{C} 26-\mathrm{C} 29$ & $1.459(3)$ \\
\hline $\mathrm{N} 2-\mathrm{C} 23$ & $1.397(2)$ & $\mathrm{C} 27-\mathrm{H} 27$ & 0.9300 \\
\hline $\mathrm{N} 2-\mathrm{C} 18$ & $1.412(2)$ & $\mathrm{C} 9-\mathrm{C} 14$ & $1.381(3)$ \\
\hline $\mathrm{N} 2-\mathrm{C} 21$ & $1.468(2)$ & $\mathrm{C} 19-\mathrm{C} 20$ & $1.381(3)$ \\
\hline $\mathrm{C} 5-\mathrm{C} 4$ & $1.390(3)$ & $\mathrm{C} 20-\mathrm{H} 20$ & 0.9300 \\
\hline $\mathrm{C} 5-\mathrm{C} 6$ & $1.411(3)$ & $\mathrm{C} 14-\mathrm{C} 13$ & $1.372(3)$ \\
\hline $\mathrm{C} 5-\mathrm{C} 10$ & $1.450(3)$ & $\mathrm{C} 14-\mathrm{H} 14$ & 0.9300 \\
\hline $\mathrm{C} 15-\mathrm{C} 16$ & $1.387(3)$ & $\mathrm{O} 1-\mathrm{C} 29$ & $1.206(3)$ \\
\hline $\mathrm{C} 15-\mathrm{C} 20$ & $1.392(3)$ & $\mathrm{C} 13-\mathrm{H} 13$ & 0.9300 \\
\hline $\mathrm{C} 15-\mathrm{C} 12$ & $1.485(3)$ & $\mathrm{C} 24-\mathrm{C} 25$ & $1.377(3)$ \\
\hline $\mathrm{C} 12-\mathrm{C} 11$ & $1.388(3)$ & $\mathrm{C} 24-\mathrm{H} 24$ & 0.9300 \\
\hline $\mathrm{C} 12-\mathrm{C} 13$ & $1.410(3)$ & $\mathrm{C} 6-\mathrm{C} 1$ & $1.392(3)$ \\
\hline $\mathrm{C} 10-\mathrm{C} 11$ & $1.388(3)$ & C29-H29 & 0.9300 \\
\hline $\mathrm{C} 10-\mathrm{C} 9$ & $1.406(3)$ & $\mathrm{C} 4-\mathrm{C} 3$ & $1.375(3)$ \\
\hline $\mathrm{C} 17-\mathrm{C} 16$ & $1.382(3)$ & $\mathrm{C} 4-\mathrm{H} 4$ & 0.9300 \\
\hline $\mathrm{C} 17-\mathrm{C} 18$ & $1.392(3)$ & $\mathrm{C} 7-\mathrm{C} 8$ & $1.502(3)$ \\
\hline C17-H17 & 0.9300 & $\mathrm{C} 7-\mathrm{H} 7 \mathrm{~A}$ & 0.9700 \\
\hline $\mathrm{C} 18-\mathrm{C} 19$ & $1.397(3)$ & C7-H7B & 0.9700 \\
\hline $\mathrm{C} 23-\mathrm{C} 24$ & $1.400(3)$ & $\mathrm{C} 25-\mathrm{H} 25$ & 0.9300 \\
\hline $\mathrm{C} 23-\mathrm{C} 28$ & $1.413(3)$ & $\mathrm{C} 1-\mathrm{C} 2$ & $1.371(3)$ \\
\hline $\mathrm{C} 21-\mathrm{C} 22$ & $1.522(3)$ & $\mathrm{C} 1-\mathrm{H} 1$ & 0.9300 \\
\hline $\mathrm{C} 21-\mathrm{H} 21 \mathrm{~A}$ & 0.9700 & $\mathrm{C} 22-\mathrm{H} 22 \mathrm{~A}$ & 0.9600 \\
\hline $\mathrm{C} 21-\mathrm{H} 21 \mathrm{~B}$ & 0.9700 & $\mathrm{C} 22-\mathrm{H} 22 \mathrm{~B}$ & 0.9600 \\
\hline $\mathrm{C} 28-\mathrm{C} 27$ & $1.379(3)$ & $\mathrm{C} 22-\mathrm{H} 22 \mathrm{C}$ & 0.9600 \\
\hline $\mathrm{C} 16-\mathrm{H} 16$ & 0.9300 & $\mathrm{C} 3-\mathrm{C} 2$ & $1.386(3)$ \\
\hline $\mathrm{N} 1-\mathrm{C} 6$ & $1.381(3)$ & $\mathrm{C} 3-\mathrm{H} 3$ & 0.9300 \\
\hline $\mathrm{N} 1-\mathrm{C} 9$ & $1.384(3)$ & $\mathrm{C} 2-\mathrm{H} 2$ & 0.9300 \\
\hline $\mathrm{N} 1-\mathrm{C} 7$ & $1.449(3)$ & $\mathrm{C} 8-\mathrm{H} 8 \mathrm{~A}$ & 0.9600 \\
\hline $\mathrm{C} 11-\mathrm{H} 11$ & 0.9300 & $\mathrm{C} 8-\mathrm{H} 8 \mathrm{~B}$ & 0.9600 \\
\hline $\mathrm{C} 26-\mathrm{C} 25$ & $1.383(3)$ & $\mathrm{C} 8-\mathrm{H} 8 \mathrm{C}$ & 0.9600 \\
\hline $\mathrm{C} 28-\mathrm{S} 1-\mathrm{C} 19$ & $99.26(9)$ & $\mathrm{C} 20-\mathrm{C} 19-\mathrm{S} 1$ & $117.78(14)$ \\
\hline $\mathrm{C} 23-\mathrm{N} 2-\mathrm{C} 18$ & $121.66(15)$ & $\mathrm{C} 18-\mathrm{C} 19-\mathrm{S} 1$ & $120.54(15)$ \\
\hline $\mathrm{C} 23-\mathrm{N} 2-\mathrm{C} 21$ & $117.98(16)$ & $\mathrm{C} 19-\mathrm{C} 20-\mathrm{C} 15$ & $122.33(18)$ \\
\hline
\end{tabular}




\begin{tabular}{|c|c|c|c|}
\hline $\mathrm{C} 18-\mathrm{N} 2-\mathrm{C} 21$ & $118.46(15)$ & $\mathrm{C} 19-\mathrm{C} 20-\mathrm{H} 20$ & 118.8 \\
\hline $\mathrm{C} 4-\mathrm{C} 5-\mathrm{C} 6$ & $119.35(19)$ & $\mathrm{C} 15-\mathrm{C} 20-\mathrm{H} 20$ & 118.8 \\
\hline $\mathrm{C} 4-\mathrm{C} 5-\mathrm{C} 10$ & $134.10(19)$ & $\mathrm{C} 13-\mathrm{C} 14-\mathrm{C} 9$ & $118.27(19)$ \\
\hline $\mathrm{C} 6-\mathrm{C} 5-\mathrm{C} 10$ & $106.54(17)$ & $\mathrm{C} 13-\mathrm{C} 14-\mathrm{H} 14$ & 120.9 \\
\hline $\mathrm{C} 16-\mathrm{C} 15-\mathrm{C} 20$ & $115.82(18)$ & $\mathrm{C} 9-\mathrm{C} 14-\mathrm{H} 14$ & 120.9 \\
\hline $\mathrm{C} 16-\mathrm{C} 15-\mathrm{C} 12$ & $122.90(18)$ & $\mathrm{C} 14-\mathrm{C} 13-\mathrm{C} 12$ & $122.65(19)$ \\
\hline $\mathrm{C} 20-\mathrm{C} 15-\mathrm{C} 12$ & $121.28(18)$ & $\mathrm{C} 14-\mathrm{C} 13-\mathrm{H} 13$ & 118.7 \\
\hline $\mathrm{C} 11-\mathrm{C} 12-\mathrm{C} 13$ & $117.99(18)$ & $\mathrm{C} 12-\mathrm{C} 13-\mathrm{H} 13$ & 118.7 \\
\hline $\mathrm{C} 11-\mathrm{C} 12-\mathrm{C} 15$ & $122.01(17)$ & $\mathrm{C} 25-\mathrm{C} 24-\mathrm{C} 23$ & 121.09 (19) \\
\hline $\mathrm{C} 13-\mathrm{C} 12-\mathrm{C} 15$ & $119.99(18)$ & $\mathrm{C} 25-\mathrm{C} 24-\mathrm{H} 24$ & 119.5 \\
\hline $\mathrm{C} 11-\mathrm{C} 10-\mathrm{C} 9$ & $119.55(18)$ & $\mathrm{C} 23-\mathrm{C} 24-\mathrm{H} 24$ & 119.5 \\
\hline $\mathrm{C} 11-\mathrm{C} 10-\mathrm{C} 5$ & $134.33(18)$ & $\mathrm{N} 1-\mathrm{C} 6-\mathrm{C} 1$ & $129.5(2)$ \\
\hline $\mathrm{C} 9-\mathrm{C} 10-\mathrm{C} 5$ & $106.12(17)$ & $\mathrm{N} 1-\mathrm{C} 6-\mathrm{C} 5$ & $109.26(17)$ \\
\hline $\mathrm{C} 16-\mathrm{C} 17-\mathrm{C} 18$ & $121.50(18)$ & $\mathrm{C} 1-\mathrm{C} 6-\mathrm{C} 5$ & $121.24(19)$ \\
\hline $\mathrm{C} 16-\mathrm{C} 17-\mathrm{H} 17$ & 119.3 & $\mathrm{O} 1-\mathrm{C} 29-\mathrm{C} 26$ & $125.5(2)$ \\
\hline $\mathrm{C} 18-\mathrm{C} 17-\mathrm{H} 17$ & 119.3 & $\mathrm{O} 1-\mathrm{C} 29-\mathrm{H} 29$ & 117.2 \\
\hline $\mathrm{C} 17-\mathrm{C} 18-\mathrm{C} 19$ & $116.38(18)$ & $\mathrm{C} 26-\mathrm{C} 29-\mathrm{H} 29$ & 117.2 \\
\hline $\mathrm{C} 17-\mathrm{C} 18-\mathrm{N} 2$ & $122.63(17)$ & $\mathrm{C} 3-\mathrm{C} 4-\mathrm{C} 5$ & $118.8(2)$ \\
\hline $\mathrm{C} 19-\mathrm{C} 18-\mathrm{N} 2$ & $120.99(17)$ & $\mathrm{C} 3-\mathrm{C} 4-\mathrm{H} 4$ & 120.6 \\
\hline $\mathrm{N} 2-\mathrm{C} 23-\mathrm{C} 24$ & $121.61(17)$ & $\mathrm{C} 5-\mathrm{C} 4-\mathrm{H} 4$ & 120.6 \\
\hline $\mathrm{N} 2-\mathrm{C} 23-\mathrm{C} 28$ & $121.17(18)$ & $\mathrm{N} 1-\mathrm{C} 7-\mathrm{C} 8$ & $113.25(19)$ \\
\hline $\mathrm{C} 24-\mathrm{C} 23-\mathrm{C} 28$ & $117.21(18)$ & $\mathrm{N} 1-\mathrm{C} 7-\mathrm{H} 7 \mathrm{~A}$ & 108.9 \\
\hline $\mathrm{N} 2-\mathrm{C} 21-\mathrm{C} 22$ & $115.14(17)$ & $\mathrm{C} 8-\mathrm{C} 7-\mathrm{H} 7 \mathrm{~A}$ & 108.9 \\
\hline $\mathrm{N} 2-\mathrm{C} 21-\mathrm{H} 21 \mathrm{~A}$ & 108.5 & $\mathrm{~N} 1-\mathrm{C} 7-\mathrm{H} 7 \mathrm{~B}$ & 108.9 \\
\hline $\mathrm{C} 22-\mathrm{C} 21-\mathrm{H} 21 \mathrm{~A}$ & 108.5 & $\mathrm{C} 8-\mathrm{C} 7-\mathrm{H} 7 \mathrm{~B}$ & 108.9 \\
\hline $\mathrm{N} 2-\mathrm{C} 21-\mathrm{H} 21 \mathrm{~B}$ & 108.5 & $\mathrm{H} 7 \mathrm{~A}-\mathrm{C} 7-\mathrm{H} 7 \mathrm{~B}$ & 107.7 \\
\hline $\mathrm{C} 22-\mathrm{C} 21-\mathrm{H} 21 \mathrm{~B}$ & 108.5 & $\mathrm{C} 24-\mathrm{C} 25-\mathrm{C} 26$ & $121.5(2)$ \\
\hline $\mathrm{H} 21 \mathrm{~A}-\mathrm{C} 21-\mathrm{H} 21 \mathrm{~B}$ & 107.5 & $\mathrm{C} 24-\mathrm{C} 25-\mathrm{H} 25$ & 119.3 \\
\hline $\mathrm{C} 27-\mathrm{C} 28-\mathrm{C} 23$ & $120.91(19)$ & $\mathrm{C} 26-\mathrm{C} 25-\mathrm{H} 25$ & 119.3 \\
\hline $\mathrm{C} 27-\mathrm{C} 28-\mathrm{S} 1$ & $118.57(15)$ & $\mathrm{C} 2-\mathrm{C} 1-\mathrm{C} 6$ & $117.9(2)$ \\
\hline $\mathrm{C} 23-\mathrm{C} 28-\mathrm{S} 1$ & $120.23(15)$ & $\mathrm{C} 2-\mathrm{C} 1-\mathrm{H} 1$ & 121.1 \\
\hline $\mathrm{C} 17-\mathrm{C} 16-\mathrm{C} 15$ & $122.44(18)$ & $\mathrm{C} 6-\mathrm{C} 1-\mathrm{H} 1$ & 121.1 \\
\hline $\mathrm{C} 17-\mathrm{C} 16-\mathrm{H} 16$ & 118.8 & $\mathrm{C} 21-\mathrm{C} 22-\mathrm{H} 22 \mathrm{~A}$ & 109.5 \\
\hline $\mathrm{C} 15-\mathrm{C} 16-\mathrm{H} 16$ & 118.8 & $\mathrm{C} 21-\mathrm{C} 22-\mathrm{H} 22 \mathrm{~B}$ & 109.5 \\
\hline $\mathrm{C} 6-\mathrm{N} 1-\mathrm{C} 9$ & $108.37(16)$ & $\mathrm{H} 22 \mathrm{~A}-\mathrm{C} 22-\mathrm{H} 22 \mathrm{~B}$ & 109.5 \\
\hline $\mathrm{C} 6-\mathrm{N} 1-\mathrm{C} 7$ & $125.54(18)$ & $\mathrm{C} 21-\mathrm{C} 22-\mathrm{H} 22 \mathrm{C}$ & 109.5 \\
\hline $\mathrm{C} 9-\mathrm{N} 1-\mathrm{C} 7$ & $125.18(18)$ & $\mathrm{H} 22 \mathrm{~A}-\mathrm{C} 22-\mathrm{H} 22 \mathrm{C}$ & 109.5 \\
\hline $\mathrm{C} 12-\mathrm{C} 11-\mathrm{C} 10$ & $120.52(18)$ & $\mathrm{H} 22 \mathrm{~B}-\mathrm{C} 22-\mathrm{H} 22 \mathrm{C}$ & 109.5 \\
\hline $\mathrm{C} 12-\mathrm{C} 11-\mathrm{H} 11$ & 119.7 & $\mathrm{C} 4-\mathrm{C} 3-\mathrm{C} 2$ & $121.3(2)$ \\
\hline $\mathrm{C} 10-\mathrm{C} 11-\mathrm{H} 11$ & 119.7 & $\mathrm{C} 4-\mathrm{C} 3-\mathrm{H} 3$ & 119.4 \\
\hline $\mathrm{C} 25-\mathrm{C} 26-\mathrm{C} 27$ & $118.33(19)$ & $\mathrm{C} 2-\mathrm{C} 3-\mathrm{H} 3$ & 119.4 \\
\hline $\mathrm{C} 25-\mathrm{C} 26-\mathrm{C} 29$ & $119.6(2)$ & $\mathrm{C} 1-\mathrm{C} 2-\mathrm{C} 3$ & $121.4(2)$ \\
\hline $\mathrm{C} 27-\mathrm{C} 26-\mathrm{C} 29$ & $122.1(2)$ & $\mathrm{C} 1-\mathrm{C} 2-\mathrm{H} 2$ & 119.3 \\
\hline $\mathrm{C} 28-\mathrm{C} 27-\mathrm{C} 26$ & $121.00(19)$ & $\mathrm{C} 3-\mathrm{C} 2-\mathrm{H} 2$ & 119.3 \\
\hline $\mathrm{C} 28-\mathrm{C} 27-\mathrm{H} 27$ & 119.5 & $\mathrm{C} 7-\mathrm{C} 8-\mathrm{H} 8 \mathrm{~A}$ & 109.5 \\
\hline $\mathrm{C} 26-\mathrm{C} 27-\mathrm{H} 27$ & 119.5 & $\mathrm{C} 7-\mathrm{C} 8-\mathrm{H} 8 \mathrm{~B}$ & 109.5 \\
\hline $\mathrm{C} 14-\mathrm{C} 9-\mathrm{N} 1$ & $129.31(18)$ & $\mathrm{H} 8 \mathrm{~A}-\mathrm{C} 8-\mathrm{H} 8 \mathrm{~B}$ & 109.5 \\
\hline $\mathrm{C} 14-\mathrm{C} 9-\mathrm{C} 10$ & $121.02(19)$ & $\mathrm{C} 7-\mathrm{C} 8-\mathrm{H} 8 \mathrm{C}$ & 109.5 \\
\hline
\end{tabular}




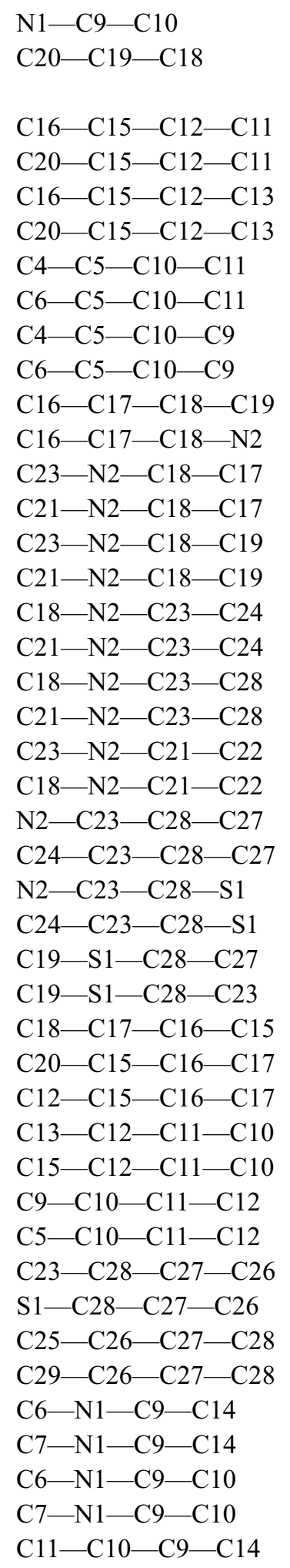

$109.67(17)$

$121.40(18)$

$21.4(3)$

-159.25 (19)

$-157.3(2)$

$22.1(3)$

$1.8(4)$

$-179.4(2)$

$-177.1(2)$

$1.7(2)$

$2.3(3)$

$-177.84(18)$

$153.75(19)$

$-10.2(3)$

$-26.4(3)$

$169.58(18)$

$-152.34(19)$

11.7 (3)

$28.8(3)$

$-167.15(17)$

$-83.2(2)$

81.4 (2)

$179.27(18)$

$0.4(3)$

$5.6(3)$

$-173.33(15)$

$153.97(16)$

$-32.19(17)$

$1.1(3)$

$-2.8(3)$

176.64 (19)

$0.0(3)$

$-178.71(18)$

0.4 (3)

$-178.4(2)$

$0.0(3)$

$173.83(16)$

$-0.6(3)$

$178.8(2)$

$-178.4(2)$

$-8.8(4)$

$1.7(2)$

$171.23(19)$

$-1.1(3)$
$\mathrm{H} 8 \mathrm{~A}-\mathrm{C} 8-\mathrm{H} 8 \mathrm{C}$

$\mathrm{H} 8 \mathrm{~B}-\mathrm{C} 8-\mathrm{H} 8 \mathrm{C}$

$\mathrm{C} 5-\mathrm{C} 10-\mathrm{C} 9-\mathrm{C} 14$

$\mathrm{C} 11-\mathrm{C} 10-\mathrm{C} 9-\mathrm{N} 1$

$\mathrm{C} 5-\mathrm{C} 10-\mathrm{C} 9-\mathrm{N} 1$

$\mathrm{C} 17-\mathrm{C} 18-\mathrm{C} 19-\mathrm{C} 20$

N2-C18-C19-C20

$\mathrm{C} 17-\mathrm{C} 18-\mathrm{C} 19-\mathrm{S} 1$

N2-C18-C19-S1

$\mathrm{C} 28-\mathrm{S} 1-\mathrm{C} 19-\mathrm{C} 20$

$\mathrm{C} 28-\mathrm{S} 1-\mathrm{C} 19-\mathrm{C} 18$

$\mathrm{C} 18-\mathrm{C} 19-\mathrm{C} 20-\mathrm{C} 15$

$\mathrm{S} 1-\mathrm{C} 19-\mathrm{C} 20-\mathrm{C} 15$

$\mathrm{C} 16-\mathrm{C} 15-\mathrm{C} 20-\mathrm{C} 19$

$\mathrm{C} 12-\mathrm{C} 15-\mathrm{C} 20-\mathrm{C} 19$

$\mathrm{N} 1-\mathrm{C} 9-\mathrm{C} 14-\mathrm{C} 13$

$\mathrm{C} 10-\mathrm{C} 9-\mathrm{C} 14-\mathrm{C} 13$

C9- $\mathrm{C} 14-\mathrm{C} 13-\mathrm{C} 12$

$\mathrm{C} 11-\mathrm{C} 12-\mathrm{C} 13-\mathrm{C} 14$

$\mathrm{C} 15-\mathrm{C} 12-\mathrm{C} 13-\mathrm{C} 14$

$\mathrm{N} 2-\mathrm{C} 23-\mathrm{C} 24-\mathrm{C} 25$

$\mathrm{C} 28-\mathrm{C} 23-\mathrm{C} 24-\mathrm{C} 25$

C9-N1-C6-C1

C7-N1-C6-C1

$\mathrm{C} 9-\mathrm{N} 1-\mathrm{C} 6-\mathrm{C} 5$

$\mathrm{C} 7-\mathrm{N} 1-\mathrm{C} 6-\mathrm{C} 5$

$\mathrm{C} 4-\mathrm{C} 5-\mathrm{C} 6-\mathrm{N} 1$

$\mathrm{C} 10-\mathrm{C} 5-\mathrm{C} 6-\mathrm{N} 1$

$\mathrm{C} 4-\mathrm{C} 5-\mathrm{C} 6-\mathrm{C} 1$

$\mathrm{C} 10-\mathrm{C} 5-\mathrm{C} 6-\mathrm{C} 1$

$\mathrm{C} 25-\mathrm{C} 26-\mathrm{C} 29-\mathrm{O} 1$

$\mathrm{C} 27-\mathrm{C} 26-\mathrm{C} 29-\mathrm{O} 1$

$\mathrm{C} 6-\mathrm{C} 5-\mathrm{C} 4-\mathrm{C} 3$

$\mathrm{C} 10-\mathrm{C} 5-\mathrm{C} 4-\mathrm{C} 3$

$\mathrm{C} 6-\mathrm{N} 1-\mathrm{C} 7-\mathrm{C} 8$

C9-N1-C7-C8

$\mathrm{C} 23-\mathrm{C} 24-\mathrm{C} 25-\mathrm{C} 26$

$\mathrm{C} 27-\mathrm{C} 26-\mathrm{C} 25-\mathrm{C} 24$

$\mathrm{C} 29-\mathrm{C} 26-\mathrm{C} 25-\mathrm{C} 24$

$\mathrm{N} 1-\mathrm{C} 6-\mathrm{C} 1-\mathrm{C} 2$

$\mathrm{C} 5-\mathrm{C} 6-\mathrm{C} 1-\mathrm{C} 2$

$\mathrm{C} 5-\mathrm{C} 4-\mathrm{C} 3-\mathrm{C} 2$

$\mathrm{C} 6-\mathrm{C} 1-\mathrm{C} 2-\mathrm{C} 3$

$\mathrm{C} 4-\mathrm{C} 3-\mathrm{C} 2-\mathrm{C} 1$
109.5

109.5

$178.0(2)$

$178.85(18)$

$-2.0(2)$

$-4.0(3)$

$176.20(18)$

$169.74(15)$

$-10.1(3)$

$-151.56(16)$

34.50 (18)

2.3 (3)

$-171.57(16)$

1.1 (3)

$-178.31(19)$

$-178.6(2)$

1.3 (3)

-0.9 (3)

0.2 (3)

178.9 (2)

$-179.10(19)$

-0.2 (3)

-179.4 (2)

$11.1(4)$

$-0.6(2)$

-170.09 (19)

178.28 (19)

-0.7 (2)

$-2.8(3)$

178.24 (19)

-179.7 (2)

0.9 (4)

$0.8(3)$

179.5 (2)

87.7 (3)

-80.1 (3)

-0.4 (3)

0.8 (3)

$-178.6(2)$

-178.9 (2)

2.4 (3)

1.4 (3)

-0.1 (3)

-1.9 (4) 
Hydrogen-bond geometry $\left(A,{ }^{\circ}\right)$

$\mathrm{Cg} 3$ and $\mathrm{Cg} 4$ are the centroids of the $\mathrm{C} 1-\mathrm{C} 6$ and $\mathrm{C} 9-\mathrm{C} 14$ rings, respectively.

\begin{tabular}{lllll}
\hline$D-\mathrm{H} \cdots A$ & $D-\mathrm{H}$ & $\mathrm{H} \cdots A$ & $D \cdots A$ & $D-\mathrm{H} \cdots A$ \\
\hline $\mathrm{C} 21-\mathrm{H} 21 A \cdots C g 4^{\mathrm{i}}$ & 0.97 & 2.95 & $3.596(2)$ & 125 \\
$\mathrm{C} 25-\mathrm{H} 25 \cdots C g 3^{\mathrm{ii}}$ & 0.93 & 2.96 & $3.558(2)$ & 123 \\
\hline
\end{tabular}

Symmetry codes: (i) $-x+1,-y,-z+2$; (ii) $-x+3 / 2, y+1 / 2,-z+3 / 2$. 